\title{
Multi-resonant compact nanoaperture with accessible large nearfields
}

\author{
Arif E. Cetin • Mustafa Turkmen · Serap Aksu • \\ Dordaneh Etezadi $\cdot$ Hatice Altug
}

Received: 14 July 2014/ Accepted: 16 October 2014/Published online: 31 October 2014

(C) Springer-Verlag Berlin Heidelberg 2014

\begin{abstract}
We present a compact and multi-resonant aperture system supporting large local electromagnetic fields, which is highly advantageous for applications demanding high signal-to-noise ratio. The system is composed of subwavelength $\mathrm{H}$-shaped apertures with extended arms fabricated through thin metal films on a free-standing dielectric membranes. Through finite-difference time-domain calculations, we numerically investigate the multi-resonant spectral nature of the compact system in detail, which is associated with the individual optical response of the constituting aperture elements. In order to realize the aperture systems working in the spectral region of interest, we introduce a fine-tuning mechanism of the optical responses through geometrical device parameters. Furthermore, we present a circuit analog of the system demonstrating the impedance characteristics of the plasmonic modes supported by the
\end{abstract}

A. E. Cetin · M. Turkmen - D. Etezadi · H. Altug

Electrical and Computer Engineering, Boston University,

Boston, MA 02215, USA

e-mail: hatice.altug@epfl.ch

\section{A. E. Cetin $(\bowtie) \cdot$ D. Etezadi $\cdot$ H. Altug}

Bioengineering Department, EPFL, Lausanne 1015, Switzerland

e-mail: arif.cetin@epfl.ch

\section{Turkmen}

Department of Electrical and Electronics Engineering,

Erciyes University, Kayseri 38039, Turkey

\section{S. Aksu}

Division of Materials Science and Engineering,

Boston University, Boston, MA 02215, USA

S. Aksu

Department of Biosystems Science and Engineering,

ETH Zurich, Basel 4058, Switzerland aperture arrays. The highly accessible large electromagnetic fields extending over a large volume improve the sensitivity of the nanostructured platform to the surface conditions by enhancing the overlap between biomolecules in the vicinity and the local optical fields. We also experimentally demonstrate this highly advantageous field generation capability through large absorption signal enhancements in the surface enhanced infrared absorption spectroscopy of protein bilayers.

\section{Introduction}

Plasmonic platforms have received significant attention due to their extraordinary optical properties. These properties provide groundbreaking applications, i.e., negative indexing, superlensing, electromagnetic cloaking, and nonlinear optics [1-11]. Recent advancements in nanomanufacturing technologies have enabled the realization of these platforms, utilizing subwavelength plasmonic structures in different shapes and sizes. Particularly, nanoapertures fabricated through metal films are of great interest to variety of fields, as they can concentrate and manipulate light at nanometer scale as well as generate dramatic nearfield enhancements [12]. Engineering of these apertures with multiple spectral resonances that can be tuned from visible to IR wavelength ranges paves the way for new improvements in chip-based optical devices, active filters, and optical modulators [13-17]. Large local electromagnetic fields supported by these nanoapertures improve the interaction between target biomolecules and the optical fields, which results in dramatic signal improvements in label-free surface enhanced biosensing applications, i.e., surface enhanced infrared absorption (SEIRA) spectroscopy [18-22]. 
In this paper, we propose a compact and multi-resonant system based on $\mathrm{H}$-shaped apertures with extended arms which supports easily accessible large local electromagnetic fields. The physical origin of the multi-resonant behavior, based on the individual response of the constituting aperture elements, is analyzed in detail through finite-difference time-domain (FDTD) simulations. We also investigate the dependence of the spectral response on the geometrical parameters, which enables the fine-tuning of the aperture optical response. Moreover, we utilize a circuit analog of the system showing the impedance characteristics of the plasmonic modes supported by the aperture arrays. In order to realize the aperture system through thin gold film on free-standing dielectric membranes, we introduce a high-precision and lift-off free nanofabrication method based on electron-beam lithography (EBL). Finally, we demonstrate that supporting highly accessible large local electromagnetic fields, which enables strong sensitivities to the changes in the surface conditions, the proposed aperture system yields strong absorption signals of protein vibrational signatures in the SEIRA spectroscopy experiments.

\section{Fabrication of the aperture system}

The corresponding H-shaped aperture with extended arms is schematically shown in Fig. 1a. The aperture is fabricated through a 30-nm-thick gold film deposited on an 80$\mathrm{nm}$-thick silicon nitride $(\mathrm{SiN})$ membrane. Figure $1 \mathrm{~b}-\mathrm{left}$ shows the scanning electron microscope (SEM) image of the fabricated aperture, where aperture width $W=100 \mathrm{~nm}$, height $H=600 \mathrm{~nm}$, horizontal arm length $L=1,250 \mathrm{~nm}$, and the distance between two vertical arms $D=200 \mathrm{~nm}$. SEM image on the right, showing a $4 \times 4$ aperture array with a period of $2 \mu \mathrm{m}$, demonstrates that the apertures are well-defined, and the aperture array is uniform over large areas.

In order to experimentally realize the apertures, we utilize a lift-off free nanofabrication technique [6, 23-28]. The fabrication process is summarized in Fig. 2. We start the fabrication process with a silicon wafer with a thickness of $500 \mu \mathrm{m}$, coated with 80-nm-thick SiN film deposited on both sides. (1) We first perform photolithography on a 2- $\mu$ m-thick MICROPOSIT ${ }^{\mathrm{TM}} \mathrm{S} 1818$ positive photoresist using SUSS MicroTec MA/BA6 Mask Aligner. Performing development, we realize the aperture pattern with a size of $750 \mu \mathrm{m} \times 750 \mu \mathrm{m}$ on the bottom SiN layer. (2) We then perform dry etching (using $\mathrm{SF}_{6}$ and He gases) with PlasmaTherm Reactive Ion Etcher to completely remove the SiN layer within the transferred pattern at the back side of the wafer. (3) The chips are then immersed in $\mathrm{KOH}$ solution for wet etching to selectively remove the silicon substrate under the top SiN layer. (4) Later, we perform EBL with Zeiss SUPRA 40VP using a positive resist, polymethyl methacrylate (950PMMA A5, MICROCHEM). (5) After development using methyl isobutyl ketone and isopropyl alcohol (MIBK-IPA, 1:3) solution, we perform dry etching (using $\mathrm{SF}_{6}$ and Ar gases) to remove the $\mathrm{SiN}$ layer while using PMMA as a mask. (6) Finally, we deposit $5 \mathrm{~nm}$ titanium, serving as an adhesion layer, and $30 \mathrm{~nm}$ gold with CHA electron beam evaporator.

\section{Working mechanism of the compact aperture}

In order to numerically analyze the aperture system, we perform FDTD calculations (Lumerical Solutions, Inc.). In FDTD simulations, the dielectric constants of titanium and gold are taken from Ref. [29]. For the unit cell consisting of a single aperture, periodic boundary condition is used along the $x$ - and $y$-directions (in order to realize the

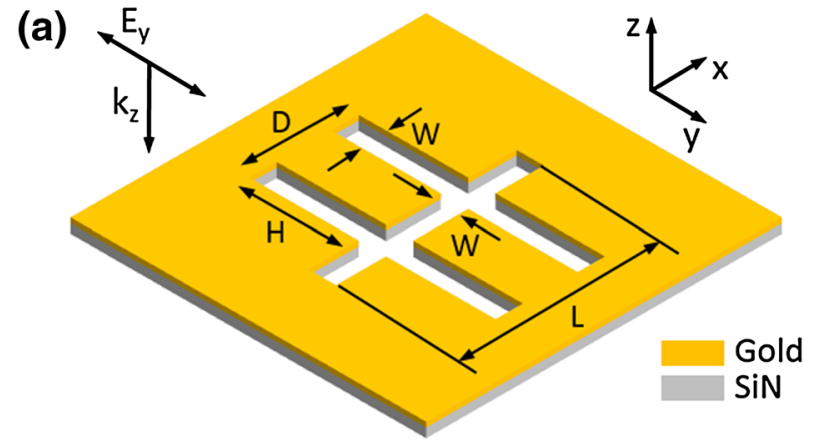

Fig. 1 a Schematic view of the H-shaped aperture with extended arms, illustrating the geometrical device parameters. Figure also shows the polarization and propagation direction of the incident light source. b SEM images of the fabricated aperture and a $4 \times 4$ aperture (b)
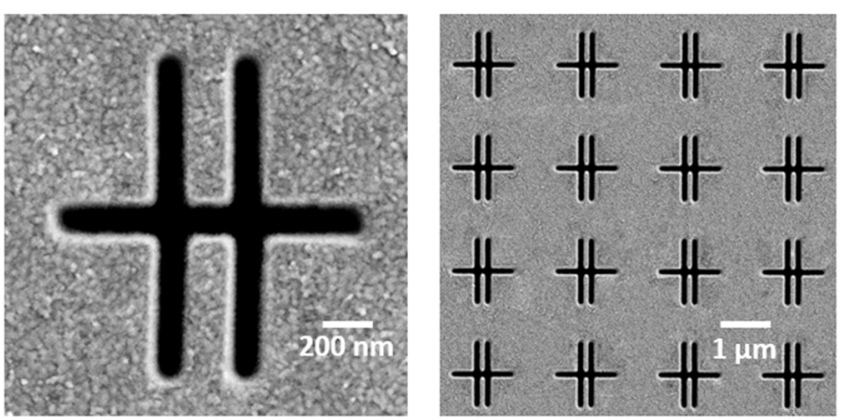

array. The corresponding device parameters are $W=100 \mathrm{~nm}$, $H=600 \mathrm{~nm}, L=1,250 \mathrm{~nm}, D=200 \mathrm{~nm}$ and the period of the aperture array is $2 \mu \mathrm{m}$. Thickness of the gold and $\mathrm{SiN}$ layers are 30 and $80 \mathrm{~nm}$, respectively 
Fig. 2 Fabrication steps of the EBL based lift-off free nanomanufacturing technique for $\mathrm{H}$-shaped apertures with extended arms

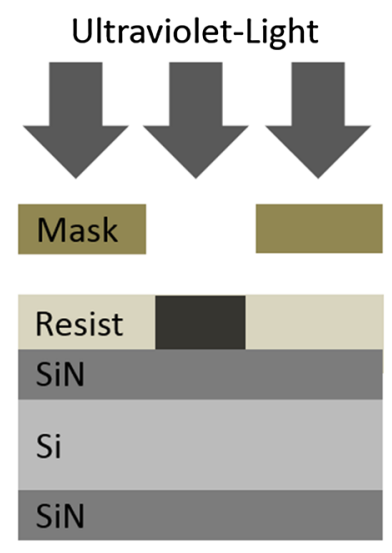

(i) Photolithography

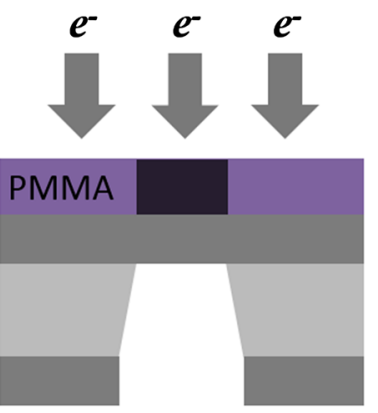

(iv) E-beam Lithography

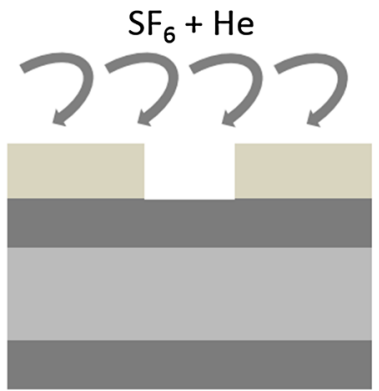

(ii) Dry Etching

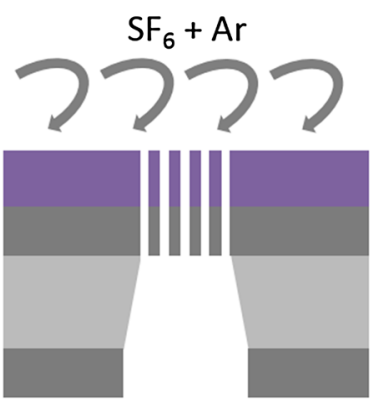

(v) Dry Etching

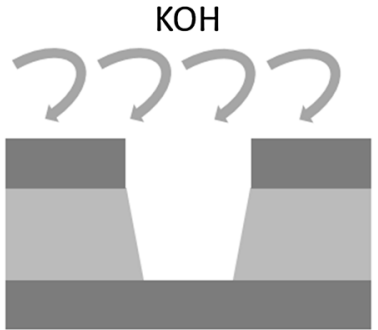

(iii) Wet Etching

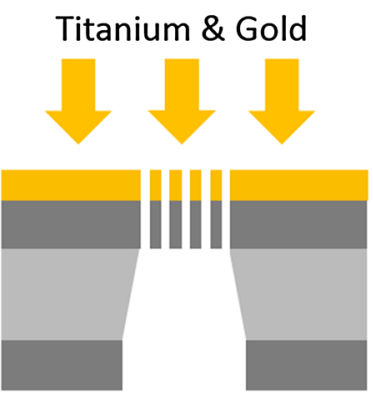

(vi) Metal Deposition periodic array effect), and perfectly matched layer boundary condition is used along the direction of the illumination source, $z$. In the simulations, the mesh size is chosen to be $2 \mathrm{~nm}$ along the $x$-, $y$ - and $z$-directions [30-32]. The numerical calculations are performed with extremely well convergence conditions with different mesh sizes, where similar near- and farfield responses are obtained. Figure $3 \mathrm{a}$ shows the calculated transmission spectrum of the system for $H=600 \mathrm{~nm}, L=1,200 \mathrm{~nm}, W=50 \mathrm{~nm}$, and $D=$ $200 \mathrm{~nm}$ under $x$ - (red line) and $y$-polarized (black line) light sources. For the $x$-polarized light source, the system supports a single resonance at $\lambda=3,703 \mathrm{~nm}$ while for the $y$-polarized source, it exhibits a multi-spectral behavior with two plasmonic modes at $\lambda_{1}=3,217 \mathrm{~nm}$ and $\lambda_{2}=5,307 \mathrm{~nm}$. The system supports higher light transmission under the $x$-polarized light source compared to the $y$-polarized one. On the other hand, for the $x$-polarization, the nearfield intensity enhancement is relatively low, i.e., the aperture system supports one order of magnitude smaller intensity enhancements compared to the case, where the system is under the $y$-polarized light source at $\lambda_{2}$. Figure $3 \mathrm{~b}$ shows the electric field intensity enhancement distribution, $|E|^{2} /\left|E_{\text {int }}\right|^{2}$ at the top surface of the metal film, for the mode at $\lambda=3,703 \mathrm{~nm}$ under the $x$-polarized source, demonstrating that the local electromagnetic fields mainly concentrate at the arms along the $y$-axis. For the $y$-polarized source, local fields mainly concentrate at the constituting aperture elements along the $x$-axis. At $\lambda_{1}$, nearfields localize at the extended arms, whereas for the mode at $\lambda_{2}$, local electromagnetic fields mainly concentrate at the junction between six arms, with more than three fold larger nearfield intensity enhancements. This strong field generation capability is highly advantageous for the applications which need high nearfield intensity enhancements such as SEIRA or Raman spectroscopy. More importantly, as opposed to the previously demonstrated nanoantenna and nanoaperture systems where the nearfields localize within a small volume (i.e., for rod antennas, they concentrate at the tip corner ends, or for bowtie apertures, they concentrate only at the bowtie gap), for the $\mathrm{H}$-shaped aperture with extended arms, the large local electromagnetic fields, corresponding to the plasmonic mode locating at $\lambda_{2}$, extend over a large area along the $x y$-plane. This nearfield characteristic is highly important for spectroscopy applications such that the sensing signals are further enhanced in the presence of the strong overlap between the optical fields and biomolecules in the vicinity, which improves the sensitivity of the system to the changes in the surface conditions. As the proposed aperture system supports a multi-spectral behavior as well as much larger nearfield 

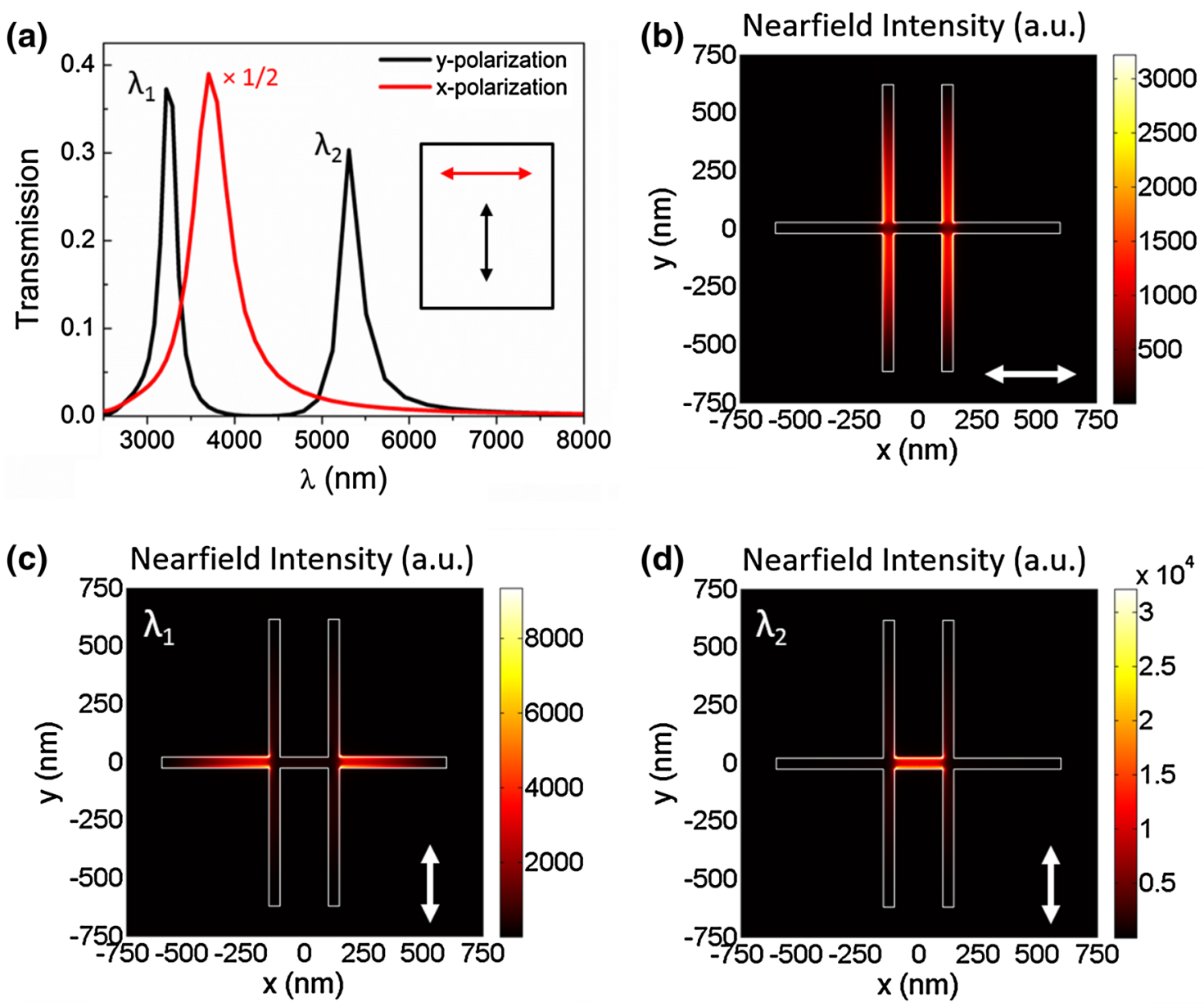

Fig. 3 a Calculated transmission spectrum of the H-shaped aperture with extended arms under the $x$ - (red line) and $y$-polarized (black line) illumination source. The polarization directions are illustrated with arrows. Nearfield intensity enhancement distribution, $|E|^{2} /\left|E_{\text {int }}\right|^{2}$ for b the $x$-polarized light source at $\lambda=3,703 \mathrm{~nm}$ and for the $y$-polarized

intensity enhancements, we investigate our system under the $y$-polarized illumination source.

The multi-resonant behavior of the compact aperture system under the $y$-polarized light source originates from the individual spectral response of the constituting aperture elements: (1) H-shaped aperture and (2) two rectangular apertures forming the extended arms. Figure 4a shows the farfield spectral response of the compact aperture (black line) and the two constituting elements. Here, the mode at $\lambda_{1}=3,217 \mathrm{~nm}$ originates from the constituting rectangular apertures $\left(\lambda_{\text {rectangular }}=2,422 \mathrm{~nm}\right)$ denoted with the blue line. Consequently, as Fig. 4b demonstrates, the compact system at $\lambda_{1}$ exhibits a similar nearfield distribution with the constituting rectangular aperture. On the other hand, the mode at $\lambda_{2}=5,307 \mathrm{~nm}$ originates from the constituting $\mathrm{H}$-shaped aperture $\left(\lambda_{\mathrm{H}-\text { shaped }}=5,506 \mathrm{~nm}\right)$ denoted with the red line. Similarly, as shown in Fig. $4 c$, the compact

light source at $\mathbf{c} \lambda_{1}=3,217 \mathrm{~nm}$ and $\mathbf{d} \lambda_{2}=5,307 \mathrm{~nm}$. The corresponding device parameters are $W=50 \mathrm{~nm}, H=600 \mathrm{~nm}$, $L=1,200 \mathrm{~nm}, D=200 \mathrm{~nm}$, and the period of the aperture array is $2 \mu \mathrm{m}$. Thickness of the gold and SiN layers are 30 and $80 \mathrm{~nm}$, respectively

system at $\lambda_{2}$ has a similar nearfield distribution with the constituting $\mathrm{H}$-shaped aperture.

Figure 5 shows the dependence of the farfield response of the compact aperture system on the geometrical parameters. Figure 5a demonstrates the variations in the transmission spectrum for different $D$ values while keeping the other parameters constant at $L=1,200 \mathrm{~nm}, H=$ $650 \mathrm{~nm}$ and $W=100 \mathrm{~nm}$ under the $y$-polarized light source. Since in the compact system, the mode at $\lambda_{2}$ originates from the constituting $\mathrm{H}$-shaped aperture, where the most of the local fields concentrate at the rectangularshaped junction between four arms, varying $D$ significantly alters this mode. Increasing $D$, extending the length of the rectangular junction along the direction normal to the polarization, allows more light to pass the aperture. Hence, the transmission of light for this mode increases with larger $D$. Transmission resonance of the rectangular apertures is 
Fig. 4 a Calculated transmission spectrum of (black line) the compact $\mathrm{H}$-shaped aperture with extended arms, (red line) the constituting $\mathrm{H}$-shaped aperture, and (blue line) the constituting extended arms under the $y$-polarized illumination light source. Nearfield intensity enhancement distribution, $|E|^{2} /$ $\left|E_{\text {int }}\right|^{2}$ for $\mathbf{b}$ the constituting extended arms at $\lambda=2,422 \mathrm{~nm}$ and $\mathbf{c}$ the constituting $\mathrm{H}$-shaped aperture at $\lambda=5,506 \mathrm{~nm}$. The polarization directions are illustrated with white arrows. The corresponding device parameters are $W=50 \mathrm{~nm}$, $H=600 \mathrm{~nm}, L=200 \mathrm{~nm}, D=$ $200 \mathrm{~nm}$ and the period of the aperture array is $2 \mu \mathrm{m}$. Thickness of the gold and SiN layers are 30 and $80 \mathrm{~nm}$, respectively
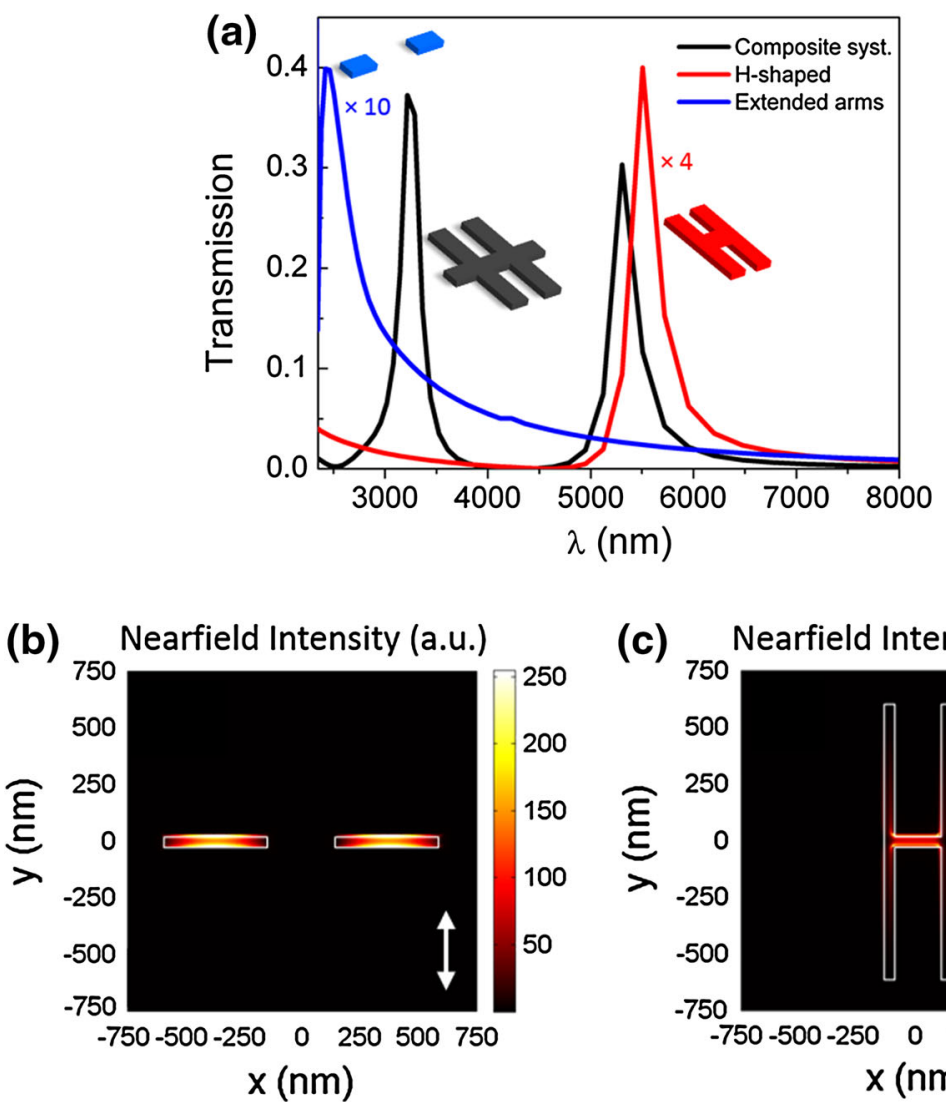

linearly proportional to the aperture length as investigated before $[33,34]$. Therefore, increasing $D$ shifts this mode to longer wavelengths. Varying $D$ also alters the mode at $\lambda_{1}$ where the local electromagnetic fields mainly concentrate at the extended rectangular-shaped arms. For the extended arms, increasing $D$ reduces the length of the rectangular aperture along the direction normal to the polarization of the illumination source. Hence, the light transmission for this mode decreases with larger $D$. For larger $D$, the mode also shifts to shorter wavelengths. Further increasing $D$ (not shown here), the compact system converges to an $\mathrm{H}$-shaped aperture such that the mode at $\lambda_{1}$ totally disappears while the one at $\lambda_{2}$ shifts to longer wavelengths. Figure $5 \mathrm{~b}$ shows the variations in the spectrum for different $H$ values while keeping the other parameters constant at $L=1,200 \mathrm{~nm}, D=200 \mathrm{~nm}$, and $W=100 \mathrm{~nm}$. Decreasing $H$, we observe negligible variations for the mode at $\lambda_{1}$. For the mode at $\lambda_{2}$, decreasing $H$, the composite system converges to a single rectangular-shaped aperture allowing more light localization at the rectangular aperture, which is perpendicular to the polarization direction. Therefore, the light transmission increases, while the mode shifts to shorter wavelengths and eventually supports a single resonance at $\lambda=3,650 \mathrm{~nm}$. Figure $5 \mathrm{c}$ shows the variations for different $L$ values while keeping the other parameters constant at $H=650 \mathrm{~nm}, D=200 \mathrm{~nm}$, and $W=100 \mathrm{~nm}$.
Varying $L$ significantly alters the mode at $\lambda_{1}$ as it mainly changes the length of the extended arms. Decreasing $L$ which shrinks the length of the extended arms in rectangular shape, the transmission for this mode decreases and the mode shifts to shorter wavelengths. Further decreasing $L$ to $200 \mathrm{~nm}$ such that the extended arms vanish (not shown here), the mode at $\lambda_{1}$ totally disappears. Consequently, the mode at $\lambda_{2}$ shifts to longer wavelengths and eventually converges to the mode of the constituting $\mathrm{H}$-shaped aperture. The light transmission for this mode also decreases and converges to the one supported by the constituting $\mathrm{H}$-shaped aperture. Figure 5d shows the variations for different $W$ values while keeping the other parameters constant at $H=650 \mathrm{~nm}, D=200 \mathrm{~nm}$, and $L=1,200 \mathrm{~nm}$. Here, we observe that for larger $W$, both modes shift to shorter wavelengths.

In order to better understand how the spectral behavior of the composite system varies with the constituting aperture elements, we use a simple circuit model schematically illustrated in Fig. 6b [35]. In the model, the impedance of the transmission lines is chosen to be characteristic impedance of the free space to effectively simulate the wave propagation within the aperture system. The load, $R_{\mathrm{L}}$, and the source resistances, $R_{\mathrm{s}}$, are matched to the characteristic impedance of the transmission lines, $Z_{0}$. In the model, the resonance wavelength of the aperture antenna is 

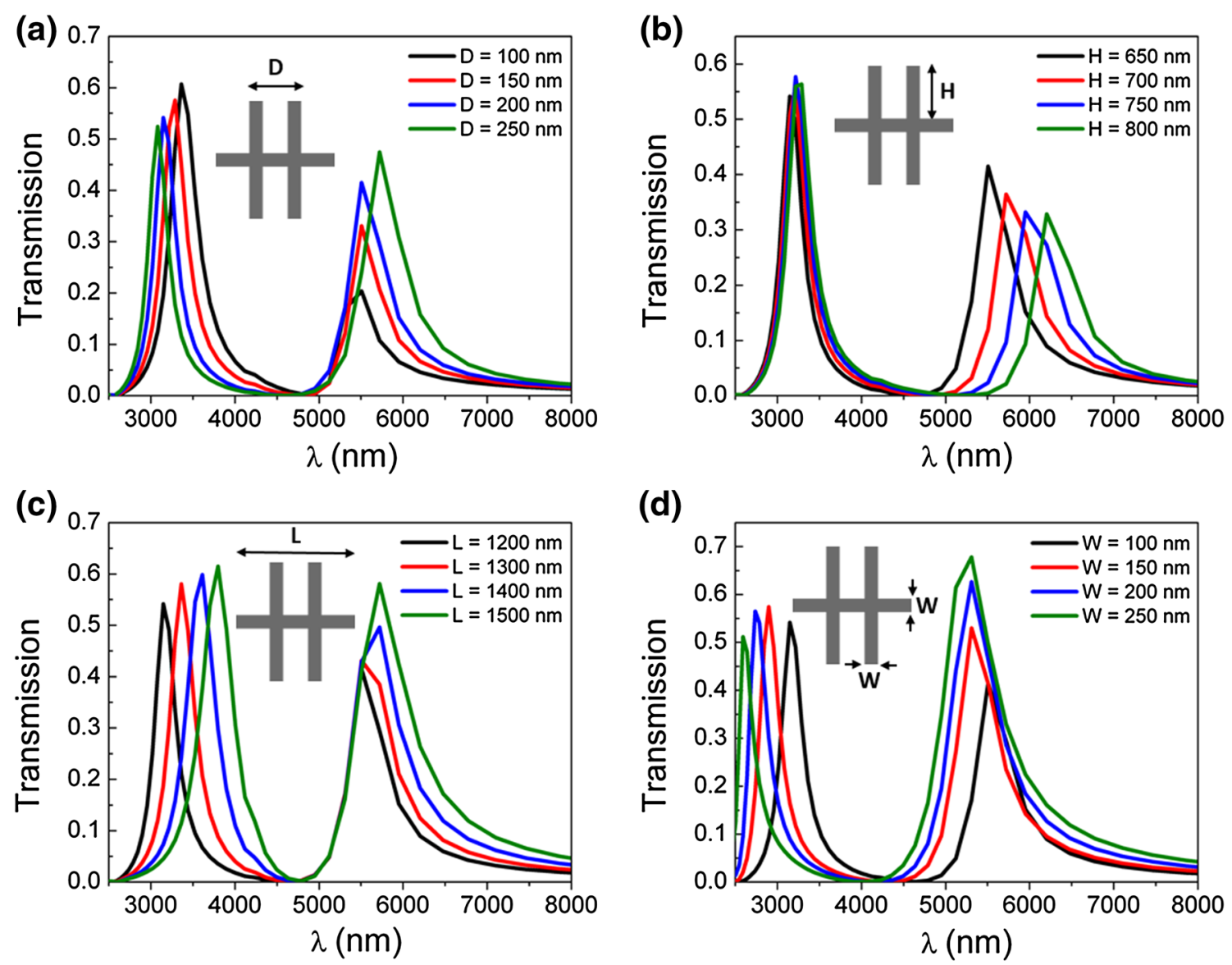

Fig. 5 Calculated transmission spectrum of the H-shaped apertures with extended arms under the $y$-polarized illumination light source for a different $D$ values at $L=1,200 \mathrm{~nm}, H=650 \mathrm{~nm}$ and $W=100 \mathrm{~nm}$, b different $H$ values at $L=1,200 \mathrm{~nm}, D=200 \mathrm{~nm}$, and $W=100 \mathrm{~nm}$,

c different $L$ values at $H=650 \mathrm{~nm}, D=200 \mathrm{~nm}$, and $W=100 \mathrm{~nm}$, d different $W$ values at $H=650 \mathrm{~nm}, D=200 \mathrm{~nm}$, and $L=1,200 \mathrm{~nm}$. The period of the aperture array is $2 \mu \mathrm{m}$. Thickness of the gold and SiN layers are 30 and $80 \mathrm{~nm}$, respectively

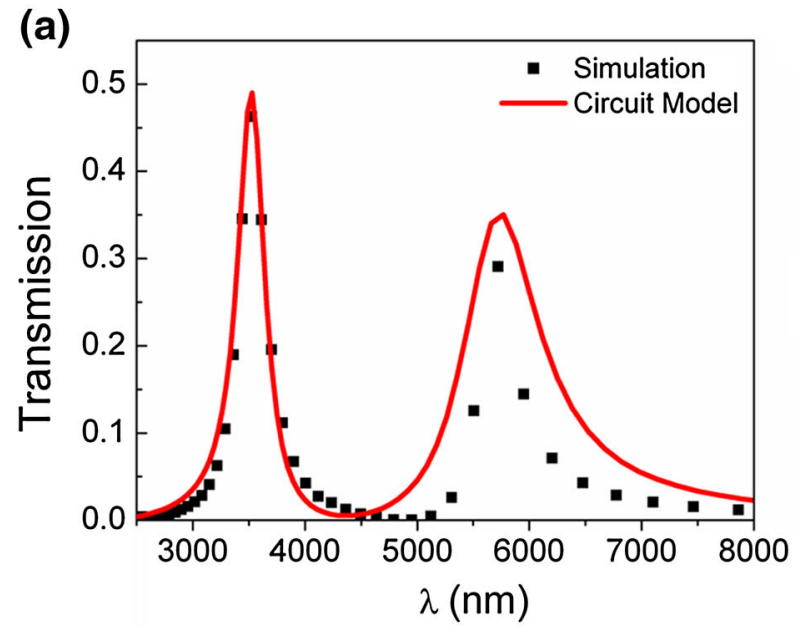

(b)

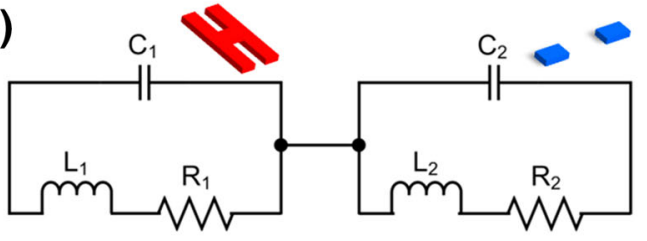

(c)

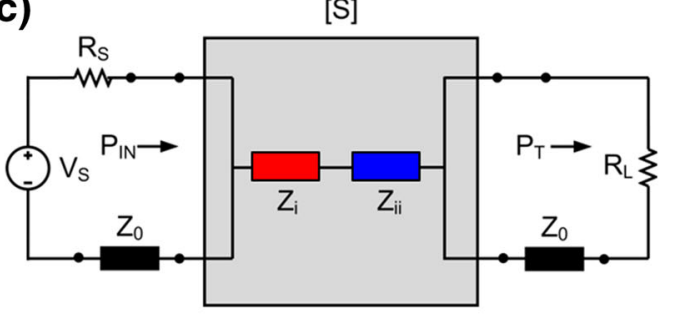

Fig. 6 a Calculated transmission spectrum of the H-shaped aperture with extended arms under the $y$-polarized illumination light source (black squares) and the circuit analog of the aperture system (red line). $\mathbf{b}$, $\mathbf{c}$ Illustration of the circuit model of the aperture system. The corresponding device parameters: (1) for the aperture system:

$H=600 \mathrm{~nm}, L=1,200 \mathrm{~nm}, W=50 \mathrm{~nm}$, and $D=200 \mathrm{~nm}$. The period of the aperture array is $2 \mu \mathrm{m}$. Thickness of the gold and SiN layers are 30 and $80 \mathrm{~nm}$, respectively. (2) For the circuit analog $C_{1}=0.23 f F, L_{1}=39.3 f H, R_{1}=1 \Omega, C_{2}=0.12 f F, L_{2}=28.6 f H$, and $R_{2}=0.03 \Omega$ 
set by the capacitor (with capacitance $=C$ ) and the inductor (with inductance $=L$ ) as $\lambda=2 \pi \sqrt{L C}$, while $R$ determines the loss. The quality factor of the circuit model then depends on the resonance wavelength and capacitance as: $Q=\pi C Z_{0} / \lambda$. When the load and source resistances are matched to the characteristic impedance of the transmission line $Z_{0}$, the transmission of the aperture system can be written as in Eq. (1).

$T=1-\left[\frac{\left|\left(Z|| Z_{0}\right)-Z_{0}\right|}{\left|\left(Z|| Z_{0}\right)+Z_{0}\right|}\right]^{2}$

Since the H-shaped aperture with extended arms is composed of two constituting aperture elements, we model the circuit analog as the two series circuits (illustrated in Fig. 6c) with impedances $Z_{i}=\left[\left(R_{1}+j \omega L_{1}\right) \|\left(j \omega C_{1}\right)^{-1}\right]$ (impedance analog of the constituting $\mathrm{H}$-shaped aperture) and $Z_{i i}=\left[\left(R_{2}+j \omega L_{2}\right) \|\left(j \omega C_{2}\right)^{-1}\right]$ (impedance analog of the constituting extended arms) such that the impedance of the composite system becomes $Z=Z_{i}+Z_{i i}$. Then, the impedance of the system can be written as in Eq. (2).

$Z=\left[\left(R_{1}+j \omega L_{1}\right) \|\left(j \omega C_{1}\right)^{-1}\right]+\left[\left(R_{2}+j \omega L_{2}\right) \|\left(j \omega C_{2}\right)^{-1}\right]$

Figure 6a shows the FDTD simulation (black squares) and the circuit model (red line) results demonstrating that our model fairly predicts the behavior of the composite aperture antenna. Here, we find the values of circuit elements as: (1) for the $\mathrm{H}$-shaped aperture, $C_{1}=0.23 \mathrm{fF}$, $L_{1}=39.3 \mathrm{fH}$, and $R_{1}=1 \Omega$ and (2) for the extended arms, $C_{2}=0.12 f F, L_{2}=28.6 f H$, and $R_{2}=0.03 \Omega$.

\section{SEIRA measurements}

We finish our discussion by a preliminary surface enhanced infrared absorption (SEIRA) spectroscopy study where the aperture system is covered with a protein bilayer consisting of protein $\mathrm{A} / \mathrm{G}$, denoted with blue in Fig. 7c-right and protein $\mathrm{IgG}$ denoted with green $(\mathrm{A} / \mathrm{G}$ from Pierce and goat IgG from Sigma). Our surface chemistry protocol ensures that the protein bilayer forms only on the gold surface as schematically illustrated in Fig. 7c-right (see methods for more detail on our surface chemistry protocols). Protein $\mathrm{A} / \mathrm{G}$ is a recombinant fusion protein which constitutes of the binding domains of both protein $A$ and protein $G$. Protein A/G sticks on the gold surface by physisorption. Bilayer formation is realized through adding the protein IgG which sticks on the protein $\mathrm{A} / \mathrm{G}$ due to the high affinity of protein $\mathrm{A} / \mathrm{G}$ to the $\mathrm{Fc}$ regions of protein $\mathrm{IgG}$ (ensuring the schematically illustrated orientation of protein $\operatorname{IgG}$ on the sensor surface). Based on the experimentally obtained complex permittivity of the protein bilayer (by the infrared reflection absorption spectroscopy), we use a Lorentz model where we fit three oscillators to the experimental data, $\varepsilon_{\text {bilayer }}=\varepsilon_{\infty}+\sum_{i=1}^{3} S_{i} /\left(\omega_{P i}^{2}-\omega^{2}-j \omega \gamma_{P i}\right)$. The values for the three oscillators are the high-frequency constant term, $\varepsilon_{\infty}=2.1901$, the oscillator resonance frequency, $\omega_{P 1}=3.12 \times 10^{14}(\mathrm{rad} / \mathrm{s}), \omega_{P 2}=2.90 \times 10^{14}(\mathrm{rad} / \mathrm{s}), \omega_{P 3}$ $=2.73 \times 10^{14}(\mathrm{rad} / \mathrm{s})$, and the damping frequency, $\gamma_{P 1}=0.98 \times 10^{13}(\mathrm{rad} / \mathrm{s}), \gamma_{P 2}=1.02 \times 10^{13}(\mathrm{rad} / \mathrm{s}), \gamma_{P 3}=$ $0.95 \times 10^{13}(\mathrm{rad} / \mathrm{s})$, and the oscillator strength, $S_{1}=2.6 \times$ $10^{27}(\mathrm{rad} / \mathrm{s})^{2}, \quad S_{2}=1.8 \times 10^{27}(\mathrm{rad} / \mathrm{s})^{2}, \quad S_{3}=0.5961 \times$ $10^{27}(\mathrm{rad} / \mathrm{s})^{2}$. The real (black line) and imaginary parts (red line) of the modeled bilayer permittivity are shown in Fig. 7a. Here, in the figure, the blue line denotes Amide I band which corresponds to $\mathrm{C}=\mathrm{O}$ stretching vibration at $\sim 6,025 \mathrm{~nm}$, and green line denotes Amide II band which corresponds to $\mathrm{C}-\mathrm{N}-\mathrm{H}$ bending and $\mathrm{C}-\mathrm{N}$ stretching vibrations at $\sim 6,562 \mathrm{~nm}$, schematically illustrated in Fig. 7c-left. For the H-shaped aperture with extended arms, as the plasmonic mode observed at the longer wavelengths $\left(\lambda_{2}\right)$ supports much larger nearfield enhancements compared to the one observed at $\lambda_{1}$, we design the aperture array with an optical response at $\lambda_{2}$ covering specifically Amide I and II vibrational bands. In the measurements, transmission response of the aperture system is collected using a Fourier transform infrared (FTIR) spectrometer (Bruker, IFS 66/s) coupled to an infrared microscope (Bruker, Hyperion 1000). The spectra are collected at a resolution of $4 \mathrm{~cm}^{-1}$ and consist of 256 scans co-added with a mirror repetition rate of $40 \mathrm{kHz}$. Spectroscopic scans are performed under a dry air purged environment to eliminate atmospheric water vapor absorption in the spectral region of interest. Here, the transmittance value is determined by taking the ratio between the light transmission through the aperture system and the light transmission through the silicon nitride membrane of the same thickness with the one used for the realization of the aperture. Figure $7 \mathrm{~b}$ shows the transmission response of the aperture spectrally locating at $\sim 5,975 \mathrm{~nm}$ (dashed black line). The device parameters of this aperture are $W=100 \mathrm{~nm}, \quad H=600 \mathrm{~nm}, \quad L=1,250 \mathrm{~nm}, \quad$ and $D=$ $200 \mathrm{~nm}$. Here, after introducing the protein layer (red line), in addition to the dips in the transmission spectrum due to the protein absorption (arising from the imaginary part of the bilayer permittivity), we also observe a red-shift due to the real part of the bilayer permittivity. In order to reliably determine the enhancement in the absorption signals for the corresponding vibrational bands while considering this redshift, we spectrally shift the bare resonance to longer wavelengths (as well as with the baseline fitting of the spectra before and after the protein bilayer addition) such that the modified bare resonance is spectrally located at $\sim 6,239 \mathrm{~nm}$ (black line) and fairly overlaps with the 
(a)

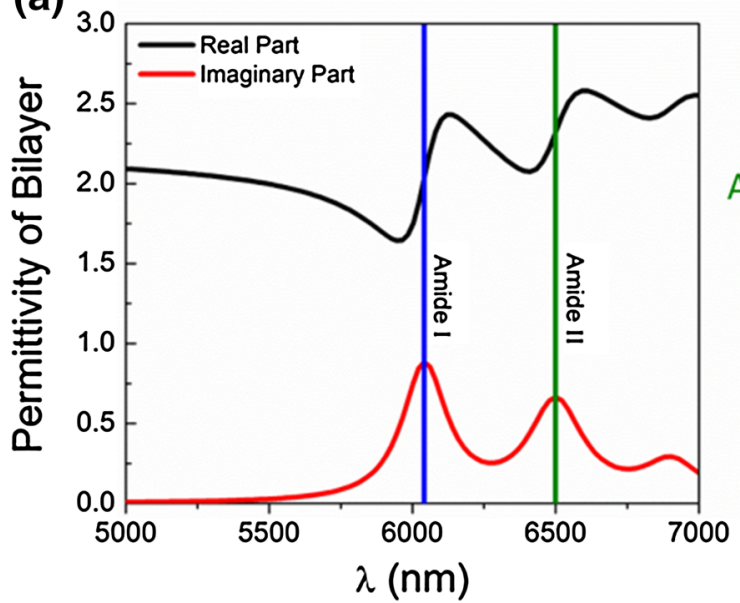

(b)

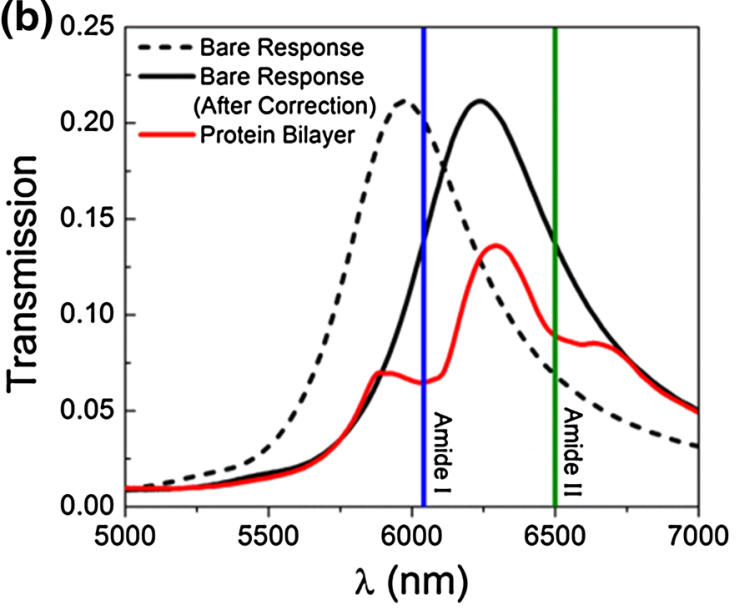

Fig. 7 a Real (black line) and imaginary (red line) parts of the permittivity of the protein bilayer (A/G + IgG) modeled using experimental results. b Experimental transmission response of the $\mathrm{H}$-shaped aperture with extended arms before (black dashed line) and after (black line) the spectral correction and after the addition of the protein bilayer (red line). In the figures, blue and green lines denote the spectral location of the Amide I and Amide II vibrational bands, respectively. The corresponding device parameters are $W=100 \mathrm{~nm}$,

response after the addition of the protein bilayer (red line). For the corresponding vibrational bands, we calculate the absorption signals $(I)$ by considering the transmission values before and after the addition of the protein bilayer, $I=\log \left(T_{\text {AfterCorrection }} / T_{\text {ProteinBilayer }}\right)$. Corresponding to the protein bilayer consisting of $\mathrm{A} / \mathrm{G}$ and $\mathrm{IgG}$, for the Amide I and II vibrational signatures, we obtain absorption signals as large as 333 and $190 \mathrm{mOD}$, respectively, which yields more than two order of magnitude signal enhancement compared to the previously presented antenna systems [32, 33]. These values are highly advantageous for SEIRA applications of biomolecules with extremely low concentrations demanding high signal-to-noise values. Figure $7 \mathrm{~d}$ shows the experimentally obtained absorption signal values (c)

\section{Vibrational Modes Protein Layer on Gold}

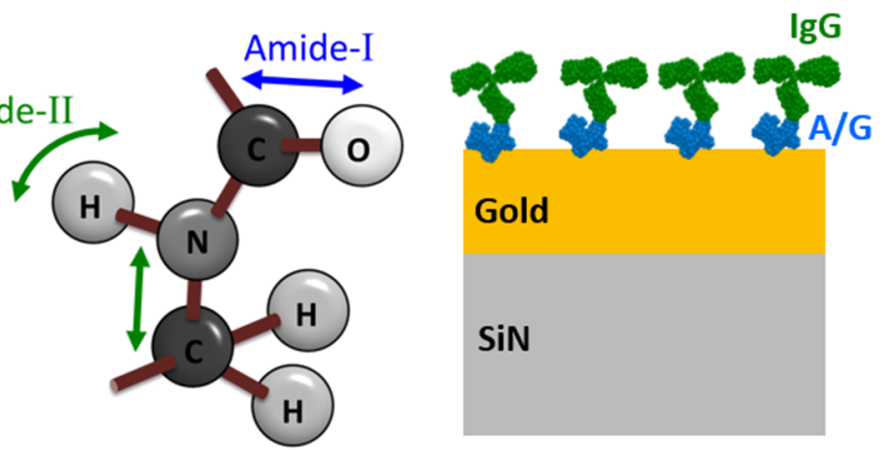

$H=600 \mathrm{~nm}, L=1,250 \mathrm{~nm}, D=200 \mathrm{~nm}$ and the period of the aperture array is $2 \mu \mathrm{m}$. Thickness of the gold and SiN layers are 30 and $80 \mathrm{~nm}$, respectively. $\mathbf{c}$ Schematic illustration of (left) the protein vibrational bands and (right) the protein bilayer covering the gold surface. d Experimentally obtained absorption signals corresponding to Amide I (blue line) and Amide II (green line) vibrational modes for the aperture systems with different $L$ values at $H=600 \mathrm{~nm}$, $D=200 \mathrm{~nm}$, and $W=100 \mathrm{~nm}$

for H-shaped apertures with extended arms for different horizontal arm lengths, $L$ while keeping the other parameters constant at $W=100 \mathrm{~nm}, \quad H=600 \mathrm{~nm}$, and $D=200 \mathrm{~nm}$. Demonstrated with FDTD calculations before, for larger $L$, the plasmonic mode located at $\lambda_{2}$ shifts to longer wavelengths. As this plasmonic mode, supported by the H-shaped aperture with extended arms, spectrally better overlaps with a particular vibrational signature ensuring the strongest interaction between local electromagnetic fields and the protein bilayer on the sensing surface, the absorption signal corresponding to that vibrational band increases. Here, this spectral overlap is considered between the modified aperture response in the presence of the red-shift due to the real part of the 
permittivity of the protein layer (instead of the bare aperture response) and the aperture response after the addition of the protein bilayer. For Amide I and II bands, maximum absorption signals are achieved at $L \sim 1,250 \mathrm{~nm}$ with 333 $\mathrm{mOD}$ and $L \sim 1,450 \mathrm{~nm}$ with $303 \mathrm{mOD}$, respectively. Here, as an additional note, the maximum Amide I absorption signal is larger compared to that of Amide II band, which can be inferred from the imaginary part of the bilayer permittivity arising from the protein absorption. In this article, we demonstrate the sensing capability of our aperture system with a spectroscopy analysis which only focuses on the plasmonic mode with the largest nearfield intensity enhancement (located at $\lambda_{2}$ ). H-shaped aperture systems with extended arms can be also designed for studying fundamental and higher order vibrational modes (secondary vibrational features) of the protein layers simultaneously using the multi-resonance character of our aperture system, i.e., multiplexed detection of Amide I and Amide III, which is out of the scope for this paper.

\section{Conclusion}

In conclusion, we introduce an $\mathrm{H}$-shaped aperture system with extended arms, which supports multi-spectral optical responses associated with easily accessible large local electromagnetic fields. The physical origin of the multiresonant behavior is investigated in detail through FDTD simulations, providing a convenient way to fine-tune the optical responses through the geometry of the constituting aperture elements. We also introduce a circuit analog of the compact system demonstrating the impedance characteristics of the plasmonic modes supported by the apertures. In order to realize the subwavelength apertures experimentally, we utilize a high-precision and lift-off free fabrication method based on EBL. Supporting highly accessible large local electromagnetic fields, the proposed aperture system yields strong absorption signals of protein vibrational modes, demonstrated through SEIRA experiments.

\subsection{Methods}

Protein Chemistry Protocols The plasmonic chip is cleaned by Nano-Strip ${ }^{\mathrm{TM}}$ from Cyantek Corporation (composed of $90 \%$ sulfuric acid, $5 \%$ peroxymonosulfuric acid, $<1 \%$ hydrogen peroxide, and $5 \%$ water) and rinsed with DIwater to dissipate any organic contamination on the surface. Then, a solution of protein $\mathrm{A} / \mathrm{G}$ in $\mathrm{Gibco}^{\circledR}$ phosphate buffered saline (PBS) $1 \times$ sterile solution-pH 7.4 is incubated. After a washing step, protein IgG is spotted on the chip surface and incubated, which is followed by a final washing step.
Acknowledgments Altug Research Group acknowledges National Science Foundation (NSF) CAREER Award and Presidential Early Career Award for Scientist and Engineers ECCS-0954790, Office of Naval Research (ONR) Young Investigator Award 11PR00755-00P00001, NSF Engineering Research Center on Smart Lighting EEC0812056 and EPFL. M. Turkmen acknowledges the Technological Research Council of Turkey (TUBITAK) under research grants and project 113E277.

\section{References}

1. L. Tong, R.R. Gattass, J.B. Ashcom, S. He, J. Lou, M. Shen, I.Z. Maxwell, J.B. Ashcom, E. Mazur, Nature 426, 816 (2003)

2. J.B. Pendry, D. Schurig, D.R. Smith, Science 312, 1780 (2006)

3. V.M. Shalaev, Nat. Photonics. 1, 41 (2007)

4. N. Engheta, Science 317, 1698 (2007)

5. A. Ourir, A. Lustrac, J.M. Lourtioz, Appl. Phys. Lett. 88, 084103 (2006)

6. M. Turkmen, S. Aksu, A.E. Cetin, A.A. Yanik, H. Altug, Opt. Express 19, 7921 (2011)

7. B. Temelkuran, M. Bayindir, E. Ozbay, R. Biswas, M.M. Sigalas, G. Tuttle, K.M. Ho, J. Appl. Phys. 87, 603 (2000)

8. S.M. Sadeghi, IEEE Trans. Nanotechnol. 10, 566 (2011)

9. P. Muhlschlegel, H.J. Eisler, O.J.F. Martin, B. Hecht, D.W. Pohl, Science 308, 1607 (2005)

10. E. Cubukcu, F. Capasso, Appl. Phys. Lett. 95, 201101 (2009)

11. E. Cubukcu, S. Zhang, Y.S. Park, G. Bartal, X. Zhang, Appl. Phys. Lett. 95, 043113 (2009)

12. S. Lal, S. Link, N.J. Halas, Nat. Photonics. 1, 641 (2007)

13. G. Leveque, O.J.F. Martin, Phys. Rev. Lett. 100, 117402 (2008)

14. J.B. Pendry, Nat. Mater. 5, 599 (2006)

15. W. Dickson, G.A. Wurtz, P.R. Evans, R.J. Pollard, A.V. Zayats, Nano Lett. 8, 281 (2008)

16. W. Dickson, G.A. Wurtz, P.R. Evans, D. O'Connor, R. Atkinson, R. Pollard, A.V. Zayats, Phys. Rev. B 76, 115411 (2007)

17. V. Zayats, R.J. Pollard, Appl. Phys. Lett. 91, 043101 (2007)

18. F. Wang, Y.R. Shen, Phys. Rev. Lett. 97, 206806 (2006)

19. H. Xu, E.J. Bjerneld, M. Kall, L. Borjesson, Phys. Rev. Lett. 83, 4357 (1999)

20. J.B. Jackson, N.J. Halas, Proc. Natl. Acad. Sci. 101, 17930 (2004)

21. A.E. Cetin, A.A. Yanik, C. Yilmaz, S. Somu, A. Busnaina, H. Altug, Appl. Phys. Lett. 98, 111110 (2011)

22. P.J. Schuck, D.P. Fromm, A. Sundaramurthy, G.S. Kino, W.E. Moerner, Phys. Rev. Lett. 94, 0174021 (2005)

23. M. Huang, A.A. Yanik, T.Y. Chang, H. Altug, Opt. Express 17, 24224 (2009)

24. A.A. Yanik, M. Huang, A. Artar, T.Y. Chang, H. Altug, Appl. Phys. Lett. 96, 021101 (2010)

25. S. Aksu, A.A. Yanik, R. Adato, A. Altar, M. Huang, H. Altug, Nano Lett. 10, 2511 (2010)

26. A.E. Cetin, A.F. Coskun, B.C. Galarreta, M. Huang, D. Herman, A. Ozcan, H. Altug, Light Sci. Appl. 3, e122 (2014)

27. A.E. Cetin, A. Mertiri, S. Erramilli, H. Altug, Adv. Opt. Mater. 1, 915 (2013)

28. A. Yanik, A.E. Cetin, M. Huang, A. Artar, S.H. Mousavi, A. Khanikaev, J.H. Connor, G. Shvets, H. Altug, Proc. Natl. Acad. Sci. 108, 11784 (2011)

29. E.D. Palik, Handbook of Optical Constants of Solids (Academic, Orlando, 1985)

30. A.E. Cetin, M. Turkmen, S. Aksu, H. Altug, IEEE Trans. Nanotechnol. 11, 208 (2011)

31. A.E. Cetin, H. Altug, ACS Nano 6, 9989 (2012)

32. A.E. Cetin, D. Etezadi, H. Altug, Adv. Opt. Mater. 2, 866 (2014)

33. R. Adato, H. Altug, Nat. Commun. 4, 2154 (2013) 
34. R. Adato, A.A. Yanik, J.J. Amsden, D.L. Kaplan, F.G. Omenetto, M.K. Hong, S. Erramilli, H. Altug, Proc. Natl. Acad. Sci. U.S.A. 106, 19227 (2009)
35. D.W. Porterfield, J.L. Hesler, R. Densing, E.R. Mueller, T.W. Crowe, R.M. Weikle II, Appl. Opt. 33, 6046 (1994) 